\title{
Occurrence of quadruple squamous cell carcinoma following allogeneic hematopoietic stem cell transplantation for leukemia: A case report
}

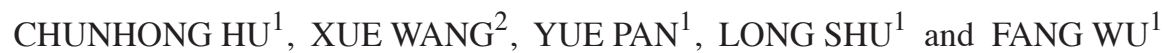 \\ ${ }^{1}$ Department of Oncology, The Second Xiangya Hospital of Central South University, Changsha, Hunan 410011; \\ ${ }^{2}$ Department of VIP Medical Services, National Cancer Center/National Clinical Research Center for Cancer/Cancer Hospital, \\ Chinese Academy of Medical Sciences and Peking Union Medical College, Beijing 100021, P.R. China
}

Received February 26, 2020; Accepted September 15, 2020

DOI: 10.3892/ol.2021.12602

\begin{abstract}
The present case study investigated a rare case of quadruple squamous cell carcinoma following allogeneic hematopoietic stem cell transplantation (HSCT) for leukemia. The main aim of the case study was to determine the pathogenesis and provide novel methods for the diagnosis and treatment of similar cases. The presence of genetic mutations in the p53, EGFR, KRAS and BRAF genes were analyzed and the presence of microsatellite instability (MSI) was determined. In addition, the expression levels of the proteins p53 and EGFR were investigated. The results identified a genetic mutation in p53, of which its expression levels were upregulated. In addition, the majority of the tumor tissues presented with MSI. Therefore, the present findings suggested that the genetic mutations in p53 caused by MSI following allogeneic HSCT may promote tumorigenesis. In addition, the expression levels of the EGFR protein were upregulated, leading to an increase in MAPK signaling pathway activation, which may also serve an important role.
\end{abstract}

\section{Introduction}

Hematopoietic stem cell transplantation (HSCT) has been identified as a potential curative treatment for leukemia $(1,2)$. However, previous studies have demonstrated that patients who develop chronic graft-versus-host disease (cGVHD) following HSCT have an increased risk of developing solid tumors, such as squamous cell carcinoma (SCC) (3-5); In a large cohort of HSCT recipients, the oral cavity is one of the most common SCC sites, accounting for $15 \%$ of all solid cancers $(6,7)$. And

Correspondence to: Dr Fang Wu, Department of Oncology, The Second Xiangya Hospital of Central South University, 139 People's Road, Changsha, Hunan 410011, P.R. China

E-mail: wufang4461@csu.edu.cn

Key words: multiple primary carcinomas, hematopoietic cell transplantation, graft-versus-host disease, p53, immune state
cGVHD is a significant risk factor independently associated with the development of secondary carcinoma $(8,9)$. This is primarily considered to occur due to the immunosuppressive therapeutic regimen, which generally includes cyclosporine, tacrolimus and corticosteroids (10). The present case report described a patient with multiorgan cGVHD, who developed quadruple SCC in the oral cavity, esophagus and skin following the prolonged exposure to combined immunosuppressant drug therapies. The main purpose of the present study was to determine its pathogenesis, and to determine whether p53, EGFR, KRAS, BRAF genes and immune status are related to tumors after HSCT, and to provide novel methods for the diagnosis and treatment of similar cases. In conclusion, the presence of microsatellite instability after HSCT indicated the instability of the genome, and might be the basis for other genetic changes. The P53 mutation might be an important promoter of multiple cancers after transplantation in the reported patient; high expression of EGFR protein increased the activity of MAPK signal transduction pathway and promoted the occurrence of tumors to a certain extent. Additionally, the long-term use of immunosuppressive agents after transplantation might promote the occurrence of tumors. Furthermore, advances in treatment and supportive care have translated into steady improvements in survival after HSCT (5). With larger numbers of long-term survivors, quantifying the late effects and related complications of transplantation has been a research priority.

\section{Case report}

Patient history. A 41-year-old male presented in September 1998 with a history of fever for 10 days, who was subsequently diagnosed with acute nonlymphocytic leukemia $\left(\mathrm{M}_{2 \mathrm{a}}\right.$ type) by bone marrow examination using Wright's and Giemsa staining (Fig. 1A). This staining was performed by incubation of samples with $100 \%$ methanol for 1-2 min, followed by incubation with the Wright stain for 4 min, working buffer for a further $4 \mathrm{~min}$ and working Giemsa stain for 4 min. The samples were then washed in distilled water and observed under a light optical microscope. The patient was treated with chemotherapy at regular intervals, which achieved a complete remission that lasted $\sim 2.5$ years. The chemotherapy regimens 

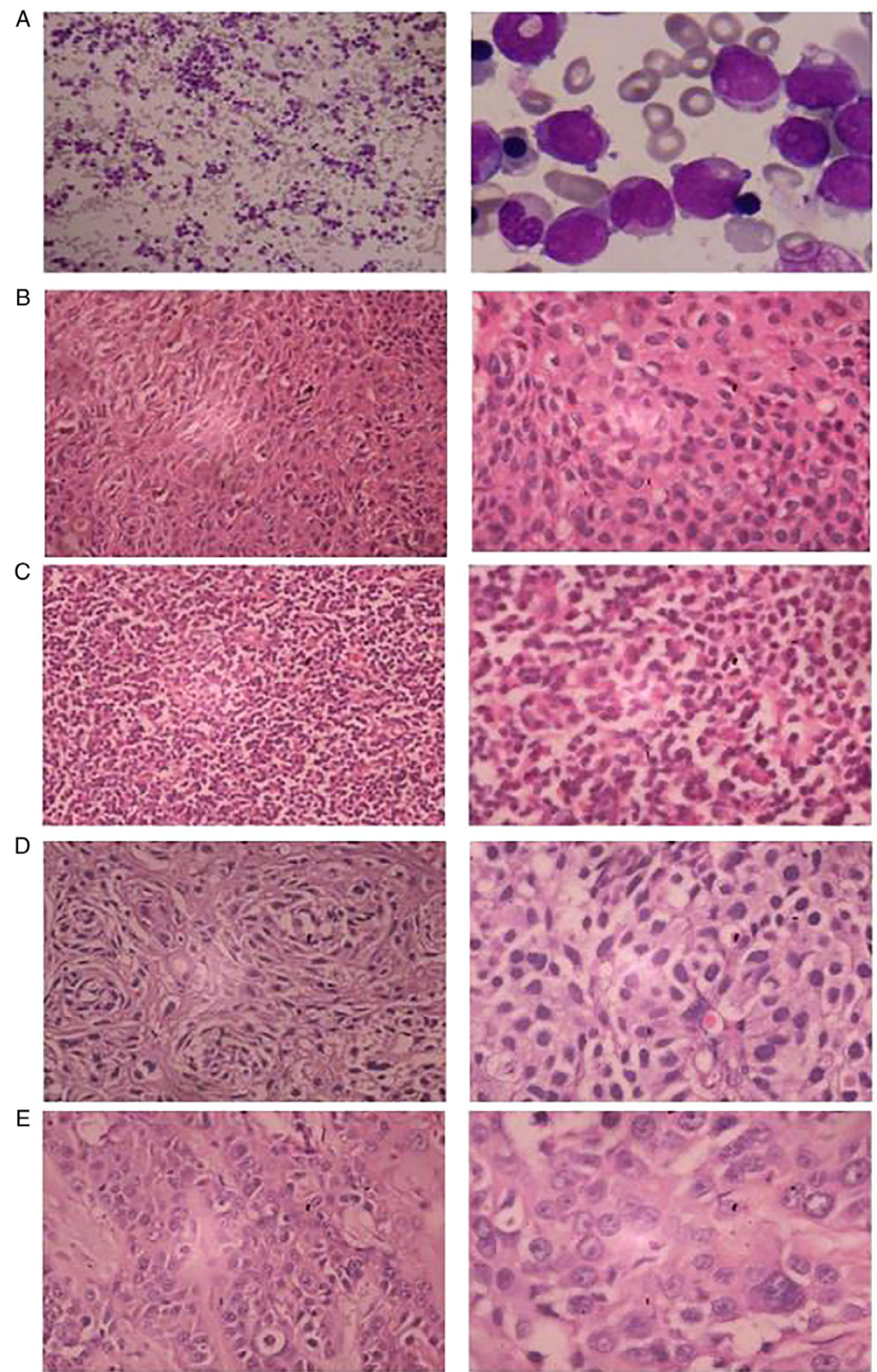

Figure 1. Histopathologic sections of quadruple carcinoma. (A) Acute nonlymphocytic leukemia $\left(\mathrm{M}_{2 \mathrm{a}}\right.$ type). Left panel represents Wright's and Giemsa staining at x100 magnification and the right panel represents Wright's and Giemsa staining at x1,000 magnification. (B) Cheilocarcinoma tissue. Left panel represents H\&E staining at x200 magnification and the right panel represents H\&E staining at x400 magnification. (C) Gingival carcinoma tissue. Left panel represents $\mathrm{H} \& \mathrm{E}$ staining at $\mathrm{x} 200$ magnification and the right panel represents $\mathrm{H} \& \mathrm{E}$ staining at $\mathrm{x} 400$ magnification. (D) Esophageal carcinoma. Left panel represents H\&E staining at x200 magnification and the right panel represents H\&E staining at x400 magnification. (E) Submaxillary gland squamous carcinoma. Left panel represents H\&E staining at x200 magnification and the right panel represents H\&E staining at x400 magnification.

included daunorubicin and cytarabine ('DA'), omacetaxine mepesuccinate, cytarabine and daunorubicin ('HAD'), omacetaxine mepesuccinate, cytarabine and vumon ('HA+Vm26'), daunorubicin, cytarabine and epirubicin ('DAE'), omacetaxine mepesuccinate, oncovin, cytarabine and prednisone ('HOAP'). The patient relapsed in August 2000, and then underwent an allogeneic peripheral blood (allo)-HSCT in Beijing from a fully human leukocyte antigen-matched male sibling. The myeloablative conditioning regimen consisted of total body irradiation, high dose cyclophosphamide and granulocyte colony-stimulating factor. Following the HSCT, cyclosporin A was prescribed to prevent GVHD prophylactically for 1 year. However, upon terminating the treatment regimen, the patient developed extensive multiorgan cGVHD, including a skin rash, stomatitis, diarrhea, Sjögren's syndrome and nephrotic syndrome, which was subsequently controlled by the combined treatment of a glucocorticoid and mycophenolate mofetil for 1.5 years. In 2004, the patient suffered condyloma acuminate, which did not recur following laser treatment

The patient was subsequently diagnosed with middle-differentiation squamous carcinoma of the right upper lip (Fig. 1B), poor-differentiation squamous carcinoma of the bottom left gum (Fig. 1C), middle-differentiation squamous carcinoma in the lower part of the esophagus (Fig. 1D) and high- and middle-differentiation squamous carcinoma of the left submaxillary gland (Fig. 1E) using H\&E staining in 
Table I. Gene mutations from cell-free tumor cells of the blood detected by denaturing high-performance liquid chromatography.

\section{A, EGFR}

\begin{tabular}{|c|c|c|}
\hline Exon & Detection result & Interpretation result \\
\hline E18 & Peak shape of the sample is same as that of the control & No mutations \\
\hline E19 & Peak shape of the sample is same as that of the control & No mutations \\
\hline E20 & Sample amplification failed & \\
\hline E21 & Peak shape of the sample is same as that of the control & No mutations \\
\hline
\end{tabular}

\section{B, BRAF}

E11 Peak shape of the sample is same as that of the control

E15 Peak shape of the sample is same as that of the control

No mutations

No mutations

\section{C, KRAS}

\begin{tabular}{lll}
\hline E2 & Peak shape of the sample is same as that of the control & No mutations \\
E3 & Peak shape of the sample is same as that of the control & No mutations \\
\hline
\end{tabular}

$\mathrm{D}, \mathrm{p} 53$

\begin{tabular}{|c|c|c|}
\hline E5 & $\begin{array}{l}64.5^{\circ} \mathrm{C} \text {, peak shape of the sample differs from that of the control; } 65.5^{\circ} \mathrm{C} \text {, it is hard } \\
\text { to judge due to insufficient sampling volume; other two temperatures, peak shape } \\
\text { of the sample is same as that of the control }\end{array}$ & Suspicious mutations \\
\hline E6 & Peak shape of the sample is same as that of the control & No mutations \\
\hline E7 & Sample amplification failed & \\
\hline E8 & Peak shape of the sample is same as that of the control & No mutations \\
\hline
\end{tabular}

the Second Xiangya Hospital of Central South University in July 2005, January 2007, October 2008 and October 2009, respectively. H\&E staining was performed on $4-\mu$ m-thick tissue sections. Specimens were stained with hematoxylin for $5 \mathrm{~min}$ and $1 \%$ eosin for $1 \mathrm{~min}$, and observed under a light optical microscope (magnifications, x200 and x400).

Each squamous carcinoma was treated with surgery and no metastasis was identified during the lymphadenectomy. The patient died in December 2009 due to postoperative pulmonary infection and malnutrition.

The patient was described as healthy beforehand, with no significant family history, and no history of smoking, or alcohol or betel nut use.

Methods. The specimens used in the following method were all from the patient who was diagnosed with quadruple SCC following allogeneic HSCT for leukemia. Cell-free DNA was extracted from the blood plasma of the peripheral blood of the patient in 2009 (since tumor cells in the peripheral blood are easier to lyse compared with normal blood cells, cell-free DNA from tumor cells are shed from the blood). Tissue samples were routinely fixed in $10 \%(\mathrm{v} / \mathrm{v})$ neutral formalin, and embedded in paraffin at a temperature of $60^{\circ} \mathrm{C}$. The paraffin-embedded sections were then cut into 3-4- $\mu$ m-thick sections, mounted on slides and then deparaffinized. To block endogenous peroxidase activity, slides were treated for 5 min at room temperature with $3 \% \mathrm{H}_{2} \mathrm{O}_{2}$, diluted in distilled water in the case of protease digestion. And the classical phenol-chloroform method was used to extract tissue DNA (11). Subsequently, PCR was performed to amplify the p53, EGFR, KRAS and BRAF genes, in addition to five microsatellite accepted loci, including D2S123, D5S346, D17S250, BAT25 and BAT26. The thermocycling conditions utilized are presented in Tables SI and SII. Denaturing high-performance liquid chromatography (DHPLC) was used to screen for mutations in the PCR amplification products of the p53, EGFR, KRAS and BRAF genes present in the blood plasma of the patient's peripheral blood (Table SIII). DHPLC was performed using the Transgenomic Wave Nucleic Acid Fragment Analysis System with a DNASep column (Transgenomic, Inc.). Triethylammonium acetate at $0.05 \%$ acetonitrile in $0.1 \mathrm{M}$ (TEAA; eluent $\mathrm{A}$ ) and $25 \%$ acetonitrile in $0.1 \mathrm{M}$ TEAA (eluent B) were comprised in the mobile phases. Using pericancerous tissues as a comparison, after surgery to remove the carcinoma of the gum, an ideal column temperature of $50^{\circ} \mathrm{C}$ was used to analyze the presence of microsatellite instability (MSI) in the patient's tumor tissue in five accepted loci (D2S123, D5S346, D17S250, BAT25 and BAT26). Then, the ABI 3730 instrument (Applied Biosystems; Thermo Fisher Scientific, Inc.) was used to sequence the p53, EGFR, KRAS and BRAF gene amplification products from the paraffin-embedded tumor tissues following the four operations. 
Table II. Gene sequencing result in tumor tissues.

\begin{tabular}{llll} 
A, Cheilocarcinoma & & & \\
\hline Gene & Exon & & Detection result \\
\hline EGFR & E18 & Normal & Normal \\
& E19 & Normal & \\
& E20 & Normal & \\
BRAF & E21 & Normal & \\
& E15 & Normal & Normal \\
KRAS & E2 & Low sequencing quality & \\
& E3 & C-A (serine-termination) \\
p53 & E6 & Normal & G-A (mutation of intron) \\
& E7 & A-T being suspicious (low mutation proportion; glutamic acid-valine)
\end{tabular}

B, Gingival carcinoma

\begin{tabular}{|c|c|c|c|}
\hline \multirow[t]{4}{*}{ EGFR } & E18 & Normal & \\
\hline & E19 & Normal & \\
\hline & E20 & C-A (isoleucine-isoleucine) & \\
\hline & $\mathrm{E} 21$ & G-A (valine-valine) & \\
\hline \multirow[t]{2}{*}{ BRAF } & E11 & Normal & \\
\hline & E15 & Normal & \\
\hline \multirow[t]{2}{*}{ KRAS } & E2 & Normal & \\
\hline & E3 & Low sequencing quality & \\
\hline \multirow[t]{5}{*}{ p53 } & E5 & G-A (leucine-leucine) & \\
\hline & & A-G (histidine-arginine) & \\
\hline & E6 & Normal & \\
\hline & E7 & Low sequencing quality, suspicious C-A & \\
\hline & E8 & A-T being suspicious (low mutation proportion; glutamic acid-valine) & \\
\hline \multicolumn{4}{|c|}{ C, Esophageal carcinoma } \\
\hline \multirow[t]{4}{*}{ EGFR } & E18 & Normal & \\
\hline & E19 & Normal & \\
\hline & E20 & Normal & \\
\hline & E21 & Normal & \\
\hline \multirow[t]{2}{*}{ BRAF } & E11 & Not yet amplify & \\
\hline & E15 & Normal & \\
\hline \multirow[t]{2}{*}{ KRAS } & E2 & Normal & \\
\hline & E3 & Low sequencing quality & \\
\hline \multirow[t]{4}{*}{ p53 } & E5 & G-A (leucine-leucine) & \\
\hline & E6 & Normal & \\
\hline & E7 & G-A & $\begin{array}{l}\text { Glutamic acid altered } \\
\text { to terminator codon }\end{array}$ \\
\hline & E8 & Normal & \\
\hline
\end{tabular}

D, Submaxillary carcinoma

\begin{tabular}{lll}
\hline EGFR & E18 & Normal \\
& E19 & Normal \\
& E20 & Normal
\end{tabular}


Table II. Continued.

D, Submaxillary carcinoma

\begin{tabular}{lll}
\hline Gene & Exon & \multicolumn{1}{c}{ Detection result } \\
\hline \multirow{3}{*}{ BRAF } & E21 & Normal \\
& E11 & Normal \\
KRAS & E15 & Normal \\
& E2 & Low sequencing quality \\
p53 & E3 & Low sequencing quality \\
& E5 & A-G (histidine-arginine) \\
& E6 & Normal \\
& E7 & Normal \\
& E8 & Normal \\
\hline
\end{tabular}

Table III. Protein expression level of p53 and EGFR in tumor tissues.

\begin{tabular}{llc}
\hline Tumor tissue (paraffin-embedded tumor tissue) & Protein expression & Detection result \\
\hline Cheilocarcinoma & EGFR & $(+\sim++)$ \\
& p53 & $(++)$ \\
Gingival carcinoma & EGFR & $(+)$ \\
& p53 & EGFR \\
Esophageal carcinoma & p53 & $(+++)$ \\
Submaxillary carcinoma & EGFR & $(+++)$ \\
& p53 & $(++)$ \\
\end{tabular}

The proportion of yellow-brown cells stained was counted and the following scale was used: i) $\leq 25 \%,(-)$; ii) $25-50 \%$, (+); iii) $50-75 \%$, (++); iv) $>75 \%,(+++)$.

Immunohistochemistry was also used to analyze the expression levels of p53 and EGFR in the tumor cells of the tissue samples. Immunohistochemistry was performed by adding $3 \%$ hydrogen peroxide solution to the tissue sections for $15 \mathrm{~min}$ at room temperature in the dark to eliminate the endogenous peroxidase activity of the tissue. Then, mouse anti-human p53 monoclonal antibody (1:50; cat.no. MAB-0674; Fuzhou Maixin Biotech Co., Ltd.), and mouse anti-human EGFR monoclonal antibody (1:50; cat. no. MAB-0196; Fuzhou Maixin Biotech Co., Ltd.) were used as primary antibodies and incubated in a humidified chamber at $4^{\circ} \mathrm{C}$ overnight. The used antibody dilution followed the guidance provided in the kit instructions. On the following day, tissue samples were incubated with the secondary biotin-labeled goat anti-rabbit antibody (UltraSensitive SP; cat. no. KIT-9709; Fuzhou Maixin Biotech Co., Ltd.), at room temperature for 15 min. Brown-yellow particles in the cell indicated positive cell staining. Each slide was counted in 5 high-power fields randomly selected under a light optical microscope (magnification, $x 400$ ), and the proportion of positive cells stained was counted as follows: i) $\leq 25 \%$, (-); ii) $25-50 \%$, (+); iii) $50-75 \%,(++)$; and iv) $>75 \%$, $(+++)$. Finally, a FACSCalibur ${ }^{\mathrm{TM}}$ flow cytometer (FACS101; BD Biosciences) was used to analyze the lymphocyte subsets present in the patient's peripheral blood to roughly estimate the patient's immune state. Analysis was performed with the CellQuest software (BD Biosciences).

\section{Results}

Genetic mutations identified in the tumor cells of the peripheral blood. For the tumor suppressor gene P53 exon E5, the sample peak shape was different from the control peak shape at $64.5^{\circ} \mathrm{C}$; at $65.5^{\circ} \mathrm{C}$, the peak shape was not easy to judge due to insufficient injection volume, and the sample exon E5 was considered to be a suspicious mutation. No mutations were found in EGFR, BRAF and KRAS genes (Table I).

Sequencing results of amplified gene products in the tumor tissues. There was a C-A mutation in the EGFR gene E20 of gingival cancer tissue. The amino acid coded before and after the mutation was the same as isoleucine, and E21 had a G-A mutation, which encodes valine before and after the mutation. None of the mutations caused any changes in gene function. No abnormality was found in the sequencing results of BRAF and KRAS (Table II).

For the sequencing of the P53 gene in cheilocarcinoma, it was found that there was a C-A mutation in E5, which converted the original codon encoding serine into a stop codon. A G-A mutation in the non-coding region of $\mathrm{E} 7$ of P53, which is an intron mutation, was observed. In the P53 gene E8 there was a 


\section{CONSOLIDATED ANALYSIS REPORT}

User: WADMIN

Date: Thu May 06 17:49:53 CST

Selection: Chromatogram

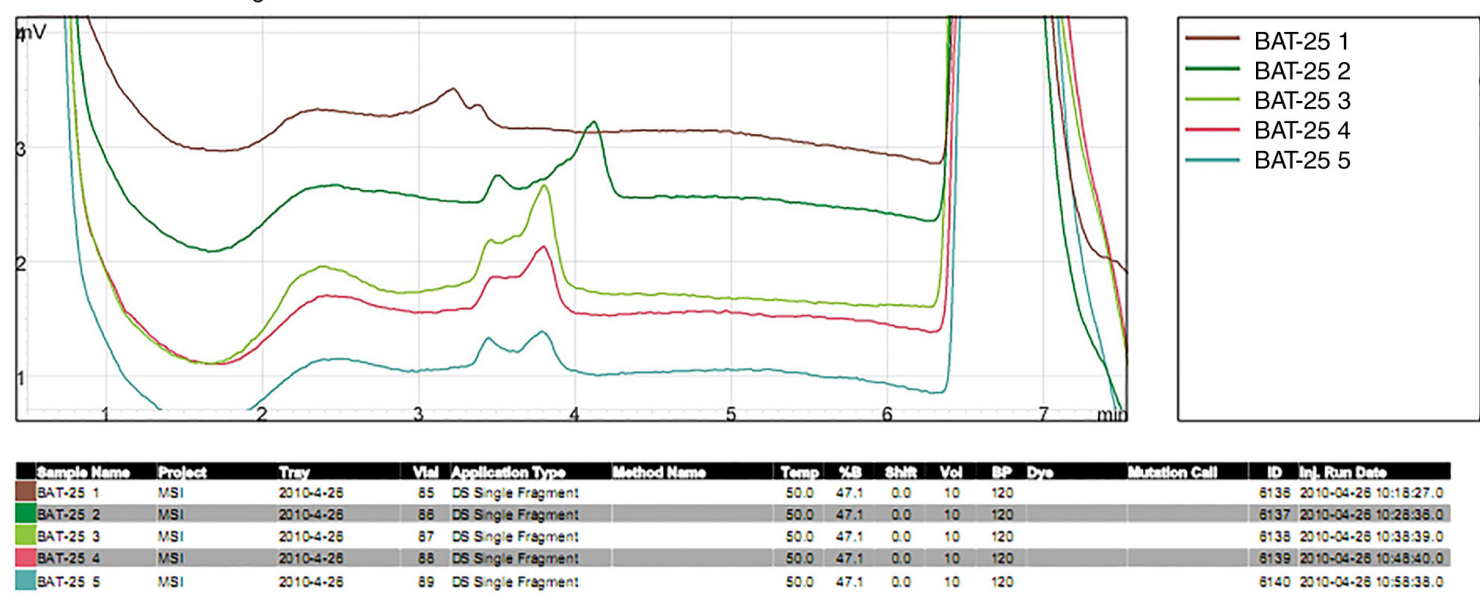

Figure 2. Presence of microsatellite instability in the tumor tissues at the BAT25 locus. 1 represents cheilocarcinoma tissue, 2 represents esophageal carcinoma, 3 represents submaxillary gland squamous carcinoma, 4 represents gingival carcinoma tissue, and 5 represents pericancerous tissues of gingival carcinoma (control).

\section{CONSOLIDATED ANALYSIS REPORT \\ User: WADMIN \\ Date: $\quad$ Thu May 06 17:54:50 CST \\ Selection: Chromatogram}
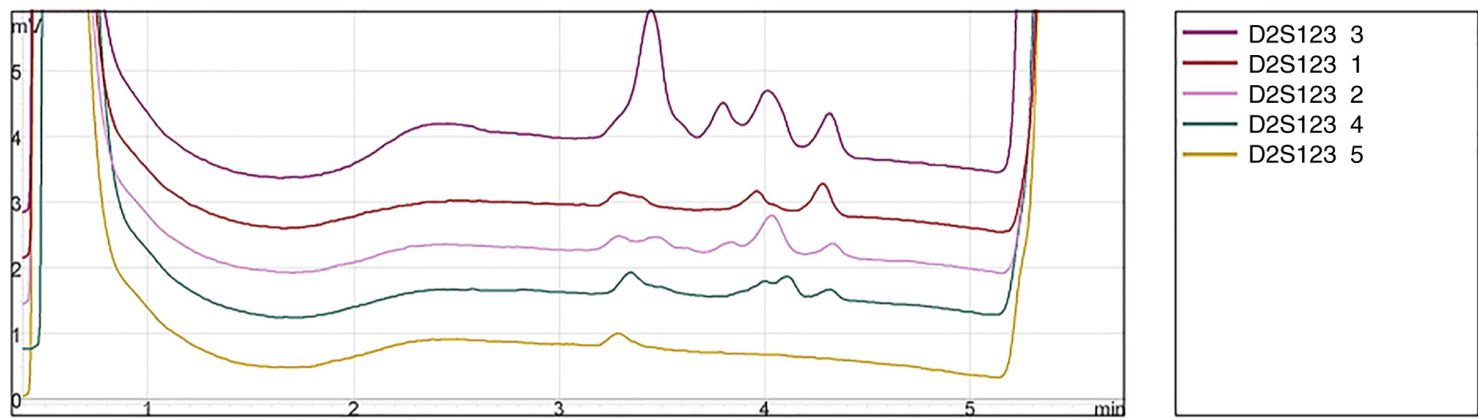

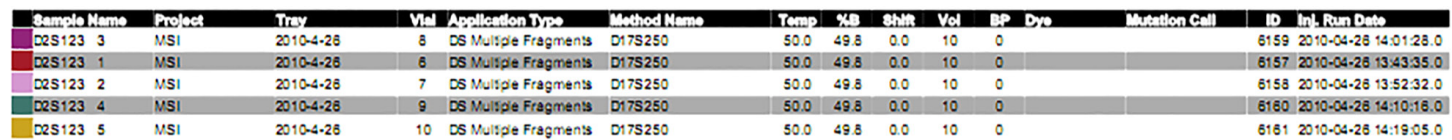

Figure 3. Presence of microsatellite instability in the tumor tissues at the D2S123 locus. 1 represents cheilocarcinoma tissue, 2 represents esophageal carcinoma, 3 represents submaxillary gland squamous carcinoma, 4 represents gingival carcinoma tissue, and 5 represents pericancerous tissues of gingival carcinoma (control).

suspicious A-T mutation in the coding region, and the coding amino acid changed from glutamic acid to valine. In gingival carcinoma tissue G-A and A-G mutations were detected in E5 of P53. The formerly coded amino acids were unchanged and both were leucine, whereas the latter changed from coding histidine to arginine. There was a suspected non-coding region C-A mutation at E7. The proportion of mutations in the coding region was low at $\mathrm{E} 8$, and $\mathrm{A}-\mathrm{T}$ mutations were suspected, the encoded amino acid was changed from glutamic acid to valine. Sequencing of the P53 gene in esophageal carcinoma tissue showed that E5 had G-A mutations, the encoded amino acids before and after the mutation were unchanged, and all were leucine. E7 had G-A mutations, which meant that the codon GAA changed to AAA, that is, glutamate changed to a stop codon. Sequencing of E5 in the P53 gene in submaxillary carcinoma tissue revealed an A-G mutation, and the encoded amino acid changed from histidine to arginine (Table II).

Protein expression levels of p53 and EGFR in the tumor tissues. The immunohistochemical results were divided into three grades according to the number of positive cells and the positive intensity. The expression levels of P53 protein in the tissues of cheilocarcinoma, gingival carcinoma and esophageal carcinoma were $(++),(+++)$ and $(+++)$, respectively. The expression levels of EGFR in cheilocarcinoma, gingival carcinoma, esophageal carcinoma and carcinoma of submaxillary were $(+\sim++),(+),(++)$ and $(++)$, respectively. Therefore, the 
CONSOLIDATED ANALYSIS REPORT

User: WADMIN

Date: $\quad$ Thu May 06 17:53:25 CST

Selection: Chromatogram

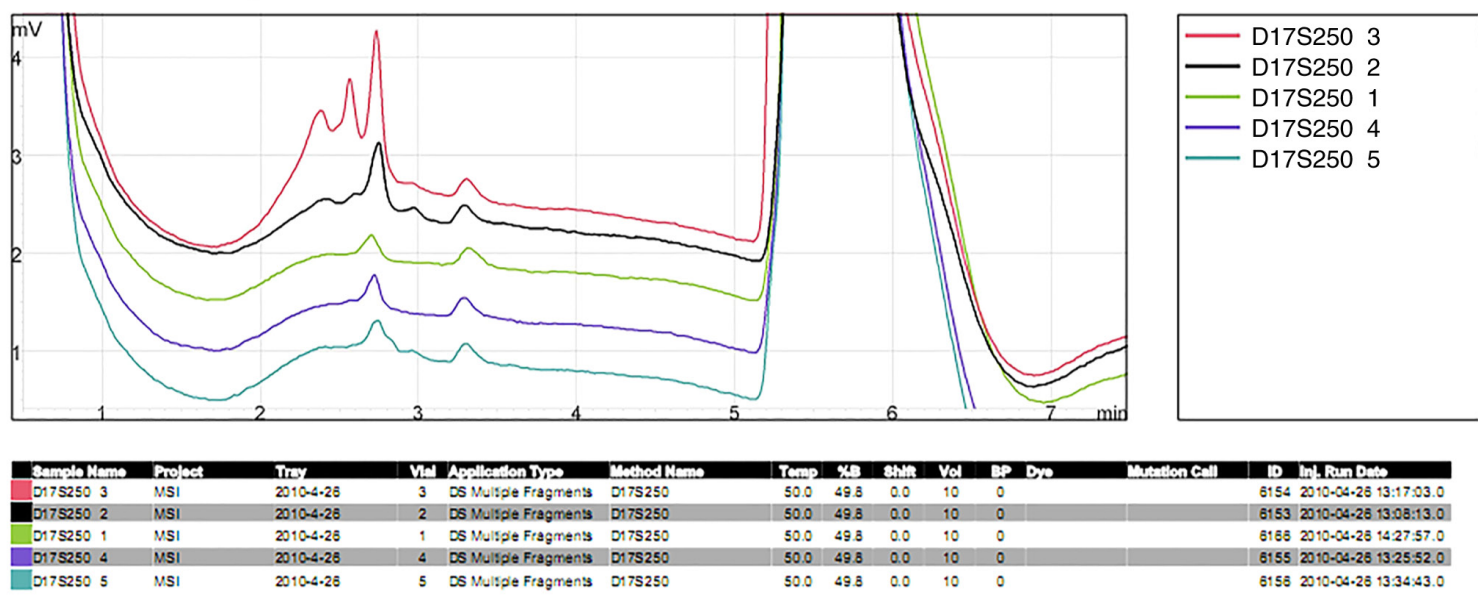

Figure 4. Presence of microsatellite instability in the tumor tissues at the D17S250 locus. 1 represents cheilocarcinoma tissue, 2 represents esophageal carcinoma, 3 represents submaxillary gland squamous carcinoma, 4 represents gingival carcinoma tissue, and 5 represents pericancerous tissues of gingival carcinoma (control).
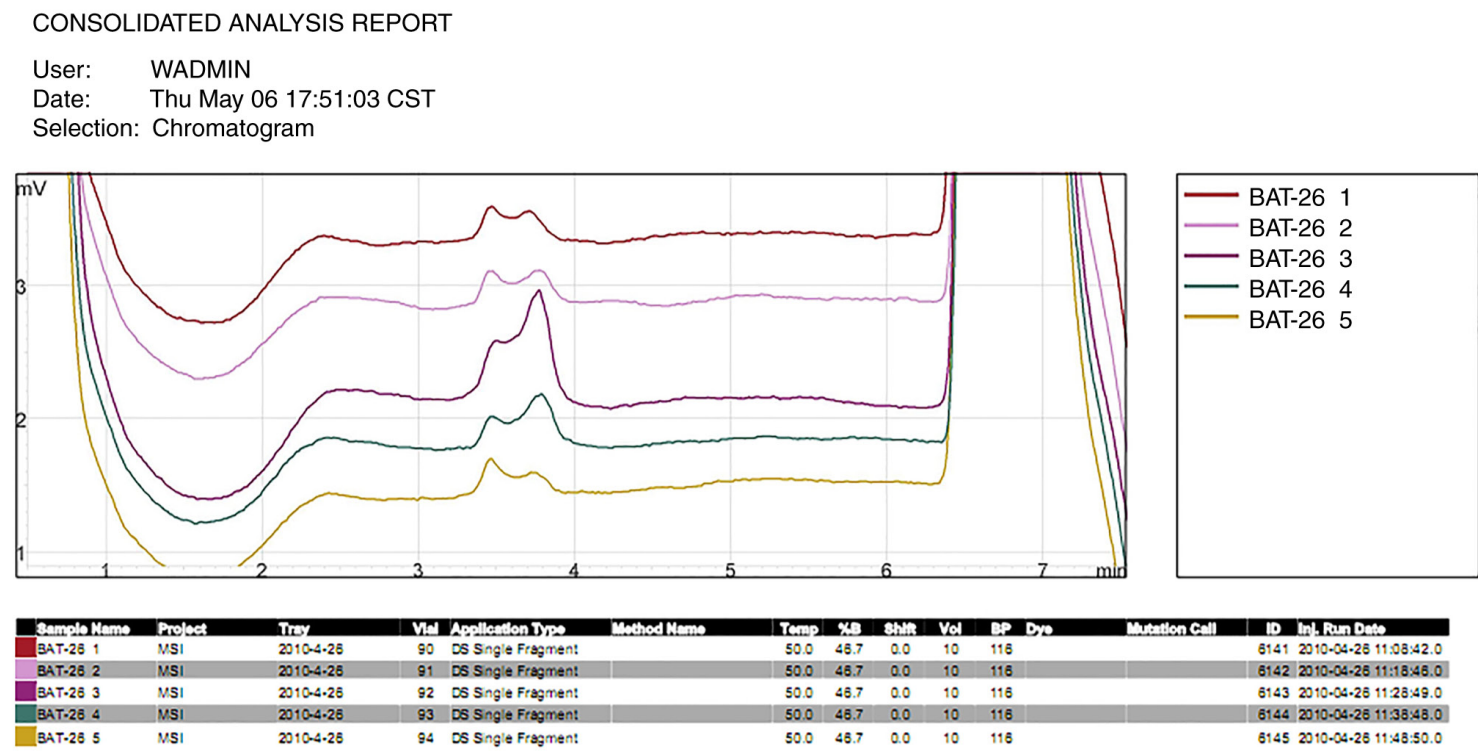

Figure 5. Presence of microsatellite instability in the tumor tissues at the BAT26 locus. 1 represents cheilocarcinoma tissue, 2 represents esophageal carcinoma, 3 represents submaxillary gland squamous carcinoma, 4 represents gingival carcinoma tissue, and 5 represents pericancerous tissues of gingival carcinoma (control).

expression levels of EGFR and P53 protein in the four tumor tissues were different (Table III).

Presence of MSI in the tumor tissues. Using pericancerous tissues of gingival carcinoma as a reference, the size of the gene segments in the cheilocarcinoma and esophageal carcinoma tissues at the BAT25 (Fig. 2) locus were reported to be altered. However, the genes in the control group at the D2S123 locus (Fig. 3) were not amplified. Notably, the length of every gene segment in the submaxillary gland carcinoma tissue differed from that of the other tumor tissues, the length of the microsatellite gene segments in the submaxillary gland carcinoma tissues were altered at the D17S250 locus (Fig. 4) and no significant differences were identified in the length of the DNA segment at the BAT26 (Fig. 5) and D5S346 (Fig. 6) loci in the four tumor tissues.

MSI was identified in cheilocarcinoma tissues at the BAT25 locus, in esophageal carcinoma tissues at the BAT25 locus and in submaxillary gland carcinoma tissues at the D2S123 and D17S250 loci; however, no abnormalities were identified in the gingival carcinoma tissues at any of the five microsatellite loci.

Identification of lymphocyte subsets in the peripheral blood. The lymphocyte subsets present in the peripheral blood were analyzed using flow cytometry. However, due to the present study being retrospective, the patient's peripheral blood was only extracted following the final operation. The results revealed the following ratio of $\mathrm{T}$ cells: i) $\mathrm{CD} 3^{+} \mathrm{T}$ cells, 
CONSOLIDATED ANALYSIS REPORT

User: WADMIN

Date: $\quad$ Thu May 06 17:51:57 CST

Selection: Chromatogram

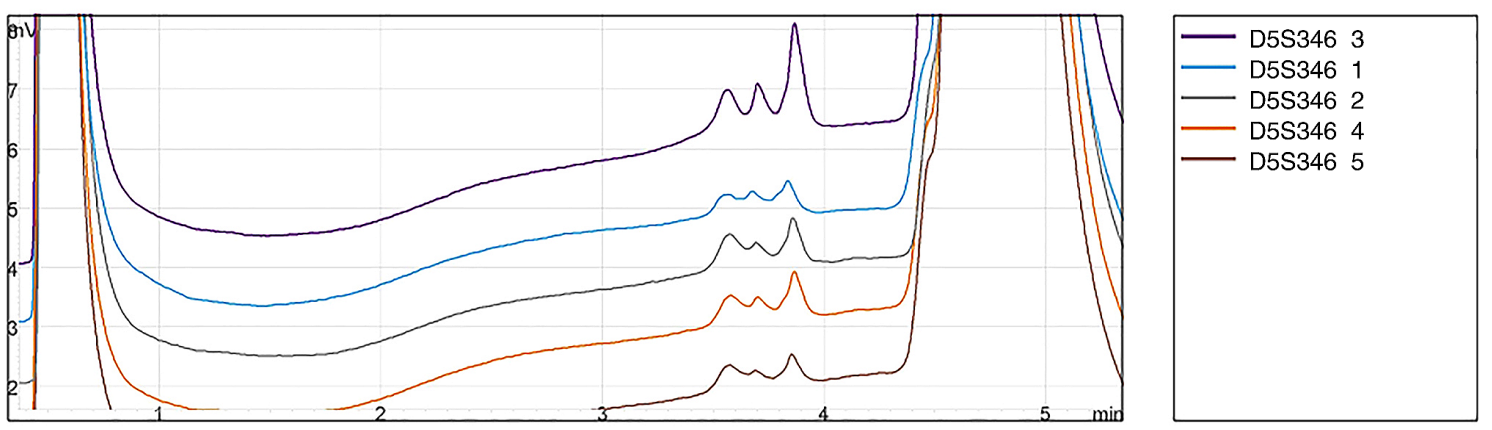

\begin{tabular}{|c|c|c|c|c|c|c|c|c|c|c|c|}
\hline enoplonms & Prober & Tres & MI & Arellementries & method:mens & Temp & $\mathbf{x}$ & sin & Yol & EP DN & I. MlRenome \\
\hline 5653463 & NSI & $2010-4 \cdot 26$ & 75 & Ds Mulspe Fragments & 065346 & 50.0 & 49.8 & 0.0 & 5 & 0 & $5148 \quad 2010 \cdot 04 \cdot 25 \quad 12: 35: 15.0$ \\
\hline DSS348 1 & MSI & $2010-4 \cdot 28$ & 73 & DS Mulsde Fragments & DES346 & 50.0 & 49.8 & 0.0 & 5 & 0 & $5146 \quad 2010-04-28 \quad 12: 19: 07.0$ \\
\hline DSS348 2 & wSI & $2010-4 \cdot 28$ & 74 & DS Wulspe Fragments & 065348 & 50.0 & 49.8 & 0.0 & 5 & 0 & $\begin{array}{lll}8147 & 2010-04-28 & 12: 27: 13.0\end{array}$ \\
\hline 0853464 & MSI & $2010-4-28$ & 78 & DS Mulspe Fragments & 065346 & 50.0 & 49.8 & 0.0 & 5 & 0 & $81492010.04-28 \quad 12: 43: 19.0$ \\
\hline Dos 348 & nSI & $2010-4 \cdot 28$ & & $\operatorname{men} 3$ & D65346 & 50.0 & 49.8 & 0.0 & & 0 & .04 \\
\hline
\end{tabular}

Figure 6. Presence of microsatellite instability in the tumor tissues at the D5S346 locus. 1 represents cheilocarcinoma tissue, 2 represents esophageal carcinoma, 3 represents submaxillary gland squamous carcinoma, 4 represents gingival carcinoma tissue, and 5 represents pericancerous tissues of gingival carcinoma (control).

$56 \%(55-84 \%)$; ii) $\mathrm{CD}^{+} \mathrm{T}$ cells, $29 \%$ (13-41\%); iii) $\mathrm{CD}^{+}$ $\mathrm{T}$ cells, $26 \%$ (31-60\%); and iv) $\mathrm{CD}^{+} / \mathrm{CD}^{+}$ratio, 0.9 (1.4-2.5), thus the ratio was decreased.

\section{Discussion}

There has been a number of reported cases $(12,13)$ of multiple primary carcinomas (MPCs) in recent years. For example, Fukuda et al (14) studied p53 mutations in relation to MPCs in a case of four separate oral cancers. However, to the best of our knowledge, the present case study was the first case involving quadruple SCC following HSCT, of which the presence of multiorgan cGVHD and the prolonged exposure to immunosuppressive therapies were factors suggested to be related to the development of SCC.

Currently, according to Warren's diagnostic criterion of MPCs in 1932 (15), the following criteria can be used to diagnose a patient with rare MPCs: i) the four squamous carcinomas are of different degrees and pathomorphologies; ii) the MPCs are located at different sites and not adjacent; and iii) the onset is during an early stage, where there is less opportunity for metastasis. The risk factors for MPCs in patients with head and neck cancer include an older age, being male, and smoking and alcohol consumption (16). In addition, it has been reported that MPCs most frequently occurred in the keratinized epithelium, such as the gingiva and hard palate (17), which is consistent with the findings in the present case study. However, the patient of the present study had no history of smoking, or alcohol or betel nut use, which suggested that other risk factors may be involved. Thus, close attention was paid to the underlying mechanism.

Allogeneic HSCT is a potential curative option for numerous hematological malignancies. However, patients undergoing allografts are at an increased risk of developing a second solid cancer, in which the overall survival varies depending on the type of secondary cancer $(5,18,19)$. A previous study (20) demonstrated that being male was associated with the development of cutaneous and oral SCC following HSCT. Furthermore, the age at transplantation, exposure to radiation as part of the conditioning regimen and chronic GVHD were identified as important risk factors for invasive solid cancers $(18,21)$. Specifically, GVHD is a major complication of allogeneic HSCT, which occurs as a result of complex immunological and inflammatory interactions, and it has been demonstrated to be responsible for significant morbidity and mortality rates $(22,23)$. In addition, taking long-term immunosuppressants was discovered to be a major risk factor, especially in combination with other drugs, such as azathioprine, cyclosporin ciclosporin and hormones $(24,25)$. For instance, the patient in the present study was a male who took ciclosporin regularly for 1 year following HSCT, after which GVHD occurred soon after drug withdrawal. The patient continued to receive immunization therapy for half a year with a glucocorticoid and 1-year immunization therapy with mycophenolate. In addition, the peripheral blood lymphocyte subsets present in the patient were investigated; the results revealed that the ratio of $\mathrm{CD}^{+} / \mathrm{CD}^{+} \mathrm{T}$ cells was markedly decreased, which indicated that the patient was under a state of immunosuppression. It was previously reported that immunosuppressive conditions led to infections and the reproduction of the tumor virus, which eventually developed into malignant tumors related to said virus $(26,27)$. The patient suffered condyloma acuminate, which is an HPV infection. In addition, inflammatory and epithelial cells release reactive oxygen and nitrogen species that are capable of causing DNA damage, which has been reported to serve important roles in cancer development (28). Therefore, the patient who underwent HSCT and developed GVHD in the present study was at an increased risk of developing a secondary cancer. Nevertheless, the mechanism by which this occurred remains unclear.

Moreover, genomic alterations in the mucosal epithelium have been frequently reported following allogeneic HSCT, 
especially among tissues affected by GVHD; this occurrence is considered to contribute to the development of secondary malignancies (29). In these circumstances, MSI has been widely identified in the genome of the patient's tumor tissue, where it was suggested to underlie the basic pathology of the tumorigenic process. Furthermore, under inflammatory conditions, inflammatory and epithelial cells release reactive oxygen and nitrogen species, which are capable of causing DNA damage (28). This, it was hypothesized that the chronic stimulation of inflammation and GVHD, in addition to the imperfect DNA repair mechanism, may be associated with the presence of MSI in tissues following transplantation.

An increasing number of studies have highlighted the role of mutant p53 proteins in altering the cancer cell secretome and modifying the tumor microenvironment (30-32). Therefore, mutations in both the p53 gene and protein in tumor tissues are likely explanations for the development of the patient's secondary solid tumor. Since the publication of Knudson's two-hit hypothesis in 1993 (33), it is common knowledge that cancer is caused by the accumulation of mutations in the DNA of cells. The term loss of heterozygosity $(\mathrm{LOH})$ has gathered increasing attention for its role in malignancies, and its accumulation in different tumor suppressor genes suggests the association of $\mathrm{LOH}$ with cancer (34). In the p53 gene, exon 4 site has been discovered to have $66 \% \mathrm{LOH}$ in heterozygous individuals. From these results, it was concluded that the inactivation of the p53 gene is related to the progression of oral cancer (35). In fact, the inactivation of p53 is considered a common step in the progression of numerous types of human cancer. Yamasaki et al (36) reported a rare case of SCC in the tongue dorsum in a 69-year-old man with a history of multiple cancers, including esophageal cancer, gastric cancer and renal cell carcinoma. Germline TP53 mutations were also discovered to be risk factors for uncontrolled cell proliferation (36). In addition, p53 expression levels were identified as an independent risk factor for early oral squamous cell carcinoma with dysplastic surgical margins (37), thus additional therapeutics and close follow-ups are required for these patients. However, to the best of our knowledge, no previous studies have reported the association between p53 mutations and HSCT; it was hypothesized that the high presence of MSI following allogeneic HSCT may inactivate tumor suppressor genes. Due to the universal presence of p53 gene mutations in the majority of types of tumor, it cannot be excluded that such mutations may be caused by non-transplantation factors; however, it also cannot be denied that HSCT itself, and the administration of immunosuppressive therapy before and after HSCT may promote the development of the tumor to a certain degree.

In addition, it was previously demonstrated that p53 interacted with EGFR to promote the activation of the EGFR/ERK/MMP-2 signaling pathway (38). The MAPK pathway is known to be closely associated with malignant tumors of the epithelium, and EGFR, KRAS and BRAF genes are all major effector molecules of the pathway. In fact, upregulated expression levels of EGFR have been detected in numerous types of tumor tissues (39). In the present study, no mutations were identified in cell-free tumor cells of the peripheral blood, or within the EGFR, KRAS and BRAF genes in the tumor tissue following the operation; however, immunohistochemistry analysis revealed that the EGFR protein expression levels in the tumor tissues were upregulated, which may be related to the amplification of the EGFR gene or the dysregulation of the MAPK signaling pathway. Although the results of the present study are unable to confirm that the overexpression of EGFR was a pathogenic factor for the patient, it has been suggested that the development of secondary tumors following HSCT are likely to overexpress EGFR, which may be sensitive to target-specific drug treatments.

The findings of the present and previous studies suggested that a frequent and thorough oral and skin examination should be recommended for all long-term HSCT survivors, with increased attention provided to those individuals who develop cGVHD and those on long-term cumulative immunosuppressant therapies. The primary therapeutic option for MPCs should be surgical removal, as the prognosis is improved compared with metastatic or recurrent carcinoma $(40,41)$. In addition, to decrease the number of deaths related to surgery, such as postoperative pneumonia or sudden death, nutritional support should also be provided following the operation.

In conclusion, the long-term use of immunosuppressants and chronic inflammation caused by HPV infection following HSCT may lead to DNA damage. The presence of MSI following HSCT indicated the instability of the genome, which provided reasons for the mutations found within the p53 gene, and suggested a major mechanism for the development of secondary multiple tumors following HSCT. Meanwhile, the identified upregulated protein expression levels of EGFR may increase the activity of the MAPK signaling transduction pathway, which may in turn, promote oncogenesis to a certain extent.

\section{Acknowledgements}

Not applicable.

\section{Funding}

No funding was received.

\section{Availability of data and materials}

The datasets used and/or analyzed during the present study are available from the corresponding author on reasonable request.

\section{Authors' contributions}

$\mathrm{CH}$ participated in the analysis, data interpretation and experimental design. XW performed the majority of the experiments. YP analyzed the data and wrote the manuscript. LS assisted with the experiments. FW designed the experiments and analyzed the data. All authors read and approved the final manuscript.

\section{Ethics approval and consent to participate}

The present study was approved by the Ethics Committee of The Second Xiangya Hospital of Central South University (approval no. 030).

\section{Patient consent for publication}

Written informed consent was obtained from the patient for publication of any accompanying images. 


\section{Competing interests}

The authors declare that they have no competing interests.

\section{References}

1. Copelan EA: Hematopoietic stem-cell transplantation. N Engl J Med 354: 1813-1826, 2006.

2. Kanakry CG, Fuchs EJ and Luznik L: Modern approaches to HLA-haploidentical blood or marrow transplantation. Nat Rev Clin Oncol 13: 132, 2016

3. Majhail NS, Brazauskas R, Rizzo JD, Sobecks RM, Wang Z, Horowitz MM, Bolwell B, Wingard JR and Socie G: Secondary solid cancers after allogeneic hematopoietic cell transplantation using busulfan-cyclophosphamide conditioning. Blood 117: 316-322, 2011

4. Majhail NS: Old and new cancers after hematopoietic-cell transplantation. Hematology Am Soc Hematol Educ Program 142-149, 2008.

5. Ehrhardt MJ, Brazauskas R, He W, Rizzo JD and Shaw BE: Survival of patients who develop solid tumors following hematopoietic stem cell transplantation. Bone Marrow Transplant 51: 83-88, 2016.

6. Rizzo JD, Curtis RE, Socié G, Sobocinski KA, Gilbert E, Landgren O, Travis LB, Travis WD, Flowers ME, Friedman DL, et al: Solid cancers after allogeneic hematopoietic cell transplantation. Blood 113: 1175-1183, 2009.

7. Inamoto Y, Shah NN, Savani BN, Shaw BE, Abraham AA, Ahmed IA, Akpek G, Atsuta Y, Baker KS, Basak GW, et al: Secondary solid cancer screening following hematopoietic cell transplantation. Bone Marrow Transplant 50: 1013-1023, 2015.

8. Chen MH, Chang PM, Li WY, Hsiao LT, Hong YC, Liu CY, Gau JP, Liu JH, Chen PM, Chiou TJ and Tzeng CH: High incidence of oral squamous cell carcinoma independent of HPV infection after allogeneic hematopoietic SCT in Taiwan. Bone Marrow Transplant 46: 567-572, 2011.

9. Themeli M, Petrikkos L, Waterhouse M, Bertz H, Lagadinou E, Zoumbos N, Finke J and Spyridonidis A: Alloreactive microenvironment after human hematopoietic cell transplantation induces genomic alterations in epithelium through an ROS-mediated mechanism: In vivo and in vitro study and implications to secondary neoplasia. Leukemia 24: 536-543, 2010.

10. Zhang L, Yu J and Wei W: Advance in targeted immunotherapy for graft-versus-host disease. Front Immunol 9: 1087, 2018.

11. Santella RM: Approaches to DNA/RNA Extraction and whole genome amplification. Cancer Epidemiol Biomarkers Prev 15: 1585-1587, 2006.

12. Obata T, Nakamura M, Mizumoto Y, Matsumoto T, Takakura M and Fujiwara H: Synchronous endometrioid adenocarcinomas in the uterine cervix and corpus. J Obstet Gynaecol Res 42: 1390-1394, 2016.

13. Adeyanju MA and Ilori AA: Multiple primary tumors. Niger J Clin Pract 20: 1346-1349, 2017.

14. Fukuda M, Nakatsuka T, Kusama K and Sakashita H: Patient with multiple primary carcinomas including 4 separate oral cancers: Study of p53 mutations and their implications for management. J Oral Maxillofac Surg 64: 1672-1679, 2006.

15. Warren S: Multiple primary malignant tumors. A survey of the literature and a statistical study. Am J cancer 16: 1358-1414, 1932

16. Hosokawa S, Takahashi G, Okamura J, Imai A, Mochizuki D, Takizawa Y, Yamatodani T, Misawa K and Mineta H: Risk and prognostic factors for multiple primary carcinomas in patients with head and neck cancer. Jpn J Clin Oncol 48: 124-129, 2018.

17. Li YD, Ma X, Han YL and Peng LW: Clinical features of multiple primary carcinomas of the oral cavity. Exp Ther Med 13: 634-638, 2017

18. Danylesko I and Shimoni A: Second malignancies after hematopoietic stem cell transplantation. Curr Treat Options Oncol 19: 9, 2018

19. Vajdic CM, Mayson E, Dodds AJ, O'Brien T, Wilcox L, Nivison-Smith I, Le Marsney R, Daniels B and Ashton LJ; CAST study investigators: Second cancer risk and late mortality in adult australians receiving allogeneic hematopoietic stem cell transplantation: A population-based cohort study. Biol Blood Marrow Transplant 22: 949-956, 2016.
20. Rizzo JD, Curtis RE, Socie G, Sobocinski KA, Gilbert E, Landgren O, Travis LB, Travis WD, Flowers ME, Friedman DL, et al: Solid cancers after allogeneic hematopoietic cell transplantation. Blood 113: 1175-1183, 2009.

21. Morton LM, Saber W, Baker KS, Barrett AJ, Bhatia S, Engels EA, Gadalla SM, Kleiner DE, Pavletic S and Burns LJ: National institutes of health hematopoietic cell transplantation late effects initiative: The subsequent neoplasms working group report. Biol Blood Marrow Transplant 23: 367-378, 2017.

22. Weng X, Xing Y and Cheng B: Multiple and recurrent squamous cell carcinoma of the oral cavity after graft-versus-host disease. J Oral Maxillofac Surg 75: 1899-1905, 2017.

23. Taskinen M, Ryhanen S and Vettenranta K: Graft-versus-host disease in stem cell transplantation. Duodecim 133: 251-258, 2017.

24. Chien SH, Liu CJ, Hong YC, Teng CJ, Hu YW, Shen CC, $\mathrm{Ku} \mathrm{FC}$, Chen SC, Yeh CM, Chiou TJ, et al: Use of azathioprine for graft-vs-host disease is the major risk for development of secondary malignancies after haematopoietic stem cell transplantation: A nationwide population-based study. Br J Cancer 112: 177-184, 2015.

25. Curtis RE, Metayer C, Rizzo JD, Socié G, Sobocinski KA, Flowers ME, Travis WD, Travis LB, Horowitz MM and Deeg HJ: Impact of chronic GVHD therapy on the development of squamous-cell cancers after hematopoietic stem-cell transplantation: An international case-control study. Blood 105: 3802-3811, 2005.

26. Chang HA, Armenian SH and Dellinger TH: Secondary neoplasms of the female lower genital tract after hematopoietic cell transplantation. J Natl Compr Canc Netw 16: 211-218, 2018.

27. Shanis D, Anandi P, Grant C, Bachi A, Vyas N, Merideth MA, Pophali PA, Koklanaris E, Ito S, Savani BN, et al: Risks factors and timing of genital human papillomavirus (HPV) infection in female stem cell transplant survivors: a longitudinal study. Bone Marrow Transplant 53: 78-83, 2018.

28. Kawanishi S, Ohnishi S, Ma N, Hiraku Y and Murata M: Crosstalk between DNA damage and inflammation in the multiple steps of carcinogenesis. Int J Mol Sci 18: 1808, 2017.

29. Akiyama M, Yamaoka M, Ohyama W, Yokoi K, Ashizuka S, Aizawa D, Ikegami M, Suzuki H, Ozaki K, Ida H and Yuza Y: Genetic profile and microsatellite instability in a case of secondary esophageal squamous cell carcinoma 12 years after allogeneic hematopoietic stem cell transplantation for aplastic anemia. J Pediatr Hematol Oncol 42: 302-306, 2020.

30. Cordani M, Pacchiana R, Butera G, D'Orazi G, Scarpa A and Donadelli M: Mutant p53 proteins alter cancer cell secretome and tumour microenvironment: Involvement in cancer invasion and metastasis. Cancer Lett 376: 303-309, 2016.

31. Guo G and Cui Y: New perspective on targeting the tumor suppressor p53 pathway in the tumor microenvironment to enhance the efficacy of immunotherapy. J Immunother Cancer 3: 9, 2015.

32. Lujambio A, Akkari L, Simon J, Grace D, Tschaharganeh DF Bolden JE, Zhao Z, Thapar V, Joyce JA, Krizhanovsky V and Lowe SW: Non-cell-autonomous tumor suppression by p53. Cell 153: 449-460, 2013.

33. Knudson AG: Antioncogenes and human cancer. Proc Natl Acad Sci USA 90: 10914-10921, 1993.

34. Jain K, Mohapatra T, Das P, Misra MC, Gupta SD, Ghosh M, Kabra M, Bansal VK, Kumar S, Sreenivas V and Garg PK: Sequential occurrence of preneoplastic lesions and accumulation of loss of heterozygosity in patients with gallbladder stones suggest causal association with gallbladder cancer. Ann Surg 260: 1073-1080, 2014.

35. Huang MF, Chang YC, Liao PS, Huang TH, Tsay CH and Chou MY: Loss of heterozygosity of p53 gene of oral cancer detected by exfoliative cytology. Oral Oncol 35: 296-301, 1999.

36. Yamasaki S, Tani R, Sakurai S, Toratani S and Okamoto T: Oral squamous cell carcinoma of the tongue dorsum with multiple cancer-associated mutations in the TP53 gene. Oral Oncol 109: 104774, 2020.

37. Yang XH, Ding L, Fu Y, Chen S, Zhang L, Zhang XX, Huang XF, Lu ZY, Ni YH and Hu QG: p53-positive expression in dysplastic surgical margins is a predictor of tumor recurrence in patients with early oral squamous cell carcinoma. Cancer Manag Res 11: $1465-1472,2019$. 
38. Gao J, Wang Y, Yang J, Zhang W, Meng K, Sun Y, Li Y and He QY: RNF128 promotes invasion and metastasis via the EGFR/MAPK/MMP-2 pathway in esophageal squamous cell carcinoma. Cancers (Basel) 11: 840, 2019.

39. Lin X, Wen G, Wang S, Lu H, Li C and Wang X: Expression and role of EGFR, cyclin D1 and KRAS in laryngocarcinoma tissues. Exp Ther Med 17: 782-790, 2019.

40. Mukerji AN and Wolf A: Synchronous esophageal and lung cancer. Thorac Surg Clin 28: 97-104, 2018.
41. Dai L, Yang HL, Yan WP, Liang Z, Xiong HC, Kang XZ, Yang YB, Fu H, Fan MY and Chen KN: The equivalent efficacy of multiple operations for multiple primary lung cancer and a single operation for single primary lung cancer. J Thorac Dis 8: 855-861, 2016. 